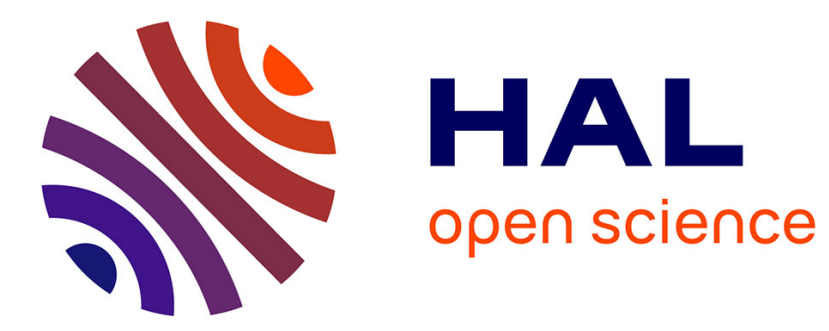

\title{
Historique de la diffraction et de la fluorescence des rayons $\mathrm{X}$
}

\author{
J. de Vries
}

\section{To cite this version:}

J. de Vries. Historique de la diffraction et de la fluorescence des rayons X. Journal de Physique IV Proceedings, 1996, 06 (C4), pp.C4-695-C4-701. 10.1051/jp4:1996467 . jpa-00254350

\section{HAL Id: jpa-00254350 https://hal.science/jpa-00254350}

Submitted on 1 Jan 1996

HAL is a multi-disciplinary open access archive for the deposit and dissemination of scientific research documents, whether they are published or not. The documents may come from teaching and research institutions in France or abroad, or from public or private research centers.
L'archive ouverte pluridisciplinaire HAL, est destinée au dépôt et à la diffusion de documents scientifiques de niveau recherche, publiés ou non, émanant des établissements d'enseignement et de recherche français ou étrangers, des laboratoires publics ou privés. 


\title{
Historique de la diffraction et de la fluorescence des rayons $\mathbf{X}$
}

\author{
J.L. de Vries
}

Philips Analytical X-Ray B.V., Lelyweg 1, 7602 EA Almelo, Pays-Bas

Résumé: Après la découverte des rayons X en 1895, il fallut attendre 1912 avant que l'expérience de von Laue montre la nature de ces rayons. Il s'agit d'ondes électromagnétiques de longueur d'onde d'environ $0,1 \mathrm{~nm}$.

La diffraction des rayons $\mathrm{X}$ a débuté par l'analyse des monocristaux pour résoudre la structure atomique, tout d'abord avec des chambres d'ionisation, puis avec des enregistrements photographiques. Par ailleurs, la spectroscopie était utilisée pour l'étude de la structure électronique selon la loi de Moseley.

L'introduction des compteurs électroniques a permis l'analyse quantitative en diffractométrie et en fluorescence. Les progrès furent ensuite très rapides, tant pour les appareils que pour les applications.

Abstract: After the discovery of X rays in 1895 it took till 1912 before the experiment by Von Laue showed these rays to be electromagnetic waves with a wave length of appr. $0.1 \mathrm{~nm}$. The diffraction of $\mathrm{X}$ rays started with analysis of single crystals to solve their structure, first with ionization chambers, later with photographic recording. Furthermore spectroscopy was used to study the electronic structure using Moseley's law.

The introduction of the electronic counters made possible the quantitative diffractometry and fluorescent spectrometry. After their introduction these methods developped very rapidly, both in instruments and applications.

\section{EVOLUTION TECHNOLOGIQUE}

Quelle était la situation de la diffraction et de la spectrométrie des rayons X en 1945 , cinquante ans après la découverte initiale?

En 1912, Von Laue demanda à un assistant de Sommerfeld, Friedrich, d'essayer une expérience pour montrer que les rayons $X$ peuvent diffracter. Le calcul admettait que la longueur d'onde des rayons $\mathrm{X}$ est du même ordre de grandeur que les distances interatomiques des cristaux.

Friedrich, assisté de Knipping a finalement, après plusieurs échecs, réussi à obtenir un diagramme de diffraction avec le spectre blanc d'un tube de rayons $\mathrm{X}$. 
Ensuite, en Angleterre, Bragg, père et fils, ont analysé des monocristaux pour établir la structure atomique. Von Laue et Bragg ont établi des formules pour calculer les directions favorables à la diffraction et la loi bien connue de Bragg est à la base de la diffractométrie et de la spectrométrie.

Debye, en 1913 à Zurich, alors qu'il étudiait la diffraction des liquides, observa qu'une poudre, comprenant un grand nombre de cristallites, irradiée par un faisceau monochromatique donne des anneaux de diffraction. La distance angulaire de ces anneaux peut être reliée, selon la loi de Bragg, aux distances interatomiques, c'est à dire les valeurs d. Ces valeurs d sont caractéristiques de la structure cristallographique des substances chimiques, qui sont ainsi identifiables. Malheureusement, à cause de la méthode photographique et des faibles intensités disponibles, une analyse rapide et quantitative n'était pas possible.

En même temps, Moseley, dans le laboratoire de Rutherford à Manchester réalisa des expériences, suivant une suggestion de Bohr, pour montrer qu'il existe une relation assez simple entre le numéro atomique $\mathrm{Z}$ d'un élément et son rayon $\mathrm{X}$ caractéristique.

C'était la base de la spectroscopie $\mathrm{X}$ et cette méthode a permis de découvrir des éléments, prévus dans la classification périodique, mais inconnus, comme le Hafnium. Des erreurs ont également été commises comme la découverte de l'élément 43, le "Masurium", alors que cet élément, ayant une durée de vie très courte, n'était pas présent.

Siegbahn en Suède effectua des études spectrométriques très précises qui conduisirent à des tables répertoriant les raies caractéristiques pour tous les éléments.

A cause de l'excitation directe, par des électrons, où l'échantillon s'évapore par chauffage, une analyse quantitative n'était pas possible. L'excitation par fluorescence était déjà suggérée par Von Hevesey et von Glockner, mais la méthode n'était pas pratique car les raies enregistrées par les méthodes photographiques étaient trop faibles.

Cette situation changea en 1945. Pendant la seconde guerre mondiale, les Etats Unis avaient besoin d'un instrument pour orienter les monocristaux de quartz avant de couper ces cristaux en tranches pour être employés dans les radars. Au M.I.T., Buerger et ses étudiants, dont Bill Parrish, ont construit un appareil pour déterminer l'orientation des tranches. Comme il faut tourner le monocristal sous la radiation directe pour trouver l'orientation où l'intensité est maximale, l'enregistrement direct est nécessaire et donc les méthodes photographiques ne peuvent plus être utilisées. Heureusement, il existait déjà un compteur électronique, avec enregistrement direct, construit par Geiger et Muller, le compteur GM. Ce système simple était commercialisé et utilisé entre autres pour analyser des poudres, mais il n'était pas possible de focaliser les rayons X. Pour cette raison, Bill Parrish a construit dans le laboratoire de Philips des Etats Unis un diffractomètre focalisant selon Bragg-Brentano. L'ensemble comprend un générateur haute tension, un goniomètre mécanique comportant l'échantillon en poudre, le compteur GM, une baie électronique et l'enregistreur. Les premiers diffractomètres étaient installés aux Etats Unis dès 1950. En réalité, la même idée était également proposée par Trost en Allemagne pendant la guerre. Ce système était alors commercialisé par une firme Allemande qui n'existe plus.

Dès que le compteur GM fut disponible, Friedman et Birks, du Naval Research Laboratory ont repris l'idée ancienne de Glockner et ont construit un prototype de spectromètre par fluorescence $\mathrm{X}$.

Les progrès de la diffraction et de la spectromètrie des rayons $\mathrm{X}$ évoluèrent différemment. La diffraction était bien connue et très employée, le diffractomètre remplaçant les films 
photographiques, était seulement une méthode plus rapide et plus quantitative, tandis que la spectrométrie était une méthode nouvelle qui cherchait sa place parmi d'autres méthodes d'analyse chimique comme la spectroscopie U.V. ou la voie humide.

\section{DIFFRACTION $X$}

En utilisant des détecteurs électroniques il faut balayer tout le domaine angulaire de diffraction de façon séquentielle. Dans ce cas, un simple générateur comme celui utilisé en travaillant avec des chambres photographiques ne suffit plus. Le débit du rayonnement devant rester constant pendant l'enregistrement, on a besoin de générateurs stabilisés, en tension et en courant. Pour gagner en intensité on peut redresser la haute tension, ou mieux, employer un système avec filtrage. La tension maximale montait progressivement de $40 \mathrm{kV}$ à $60 \mathrm{kV}$, puis $100 \mathrm{kV}$ pour la spectrométrie. Le tube à rayons X s'améliorait aussi: moins d'impuretés, une durée de vie supérieure, des fenêtres plus minces avec quatre sorties, deux avec une focale carrée et deux avec une focale linéaire.

La stabilité du débit des rayons $\mathrm{X}$ était naturellement contrôlée par les utilisateurs. Mais, pour les premiers diffractomètres munis d'un cristal courbé, permettant de réduire l'intensité diffractée trop intense pour le compteur GM, on imputa tout d'abord les fluctuations d'intensité au débit des rayons $X$, alors qu'il s'avéra que ces fluctuations étaient dues au cristal très sensible à la température, un courant d'air provenant d'une ouverture de porte suffisait à provoquer un changement des distances interréticulaires du cristal.

Le développement des détecteurs a commencé avec le compteur GM. Dans ce tube, la décharge électronique après l'absorption d'un photon X s'effectue dans le volume total du compteur. L'impulsion électronique est alors suffisamment intense pour être mesurée sans amplification. Mais le temps mort, temps nécessaire pour récupérer l'impulsion suivante est très long, rendant impossible l'enregistrement de raies d'intensités importantes. Il faut alors corriger par le temps mort les valeurs expérimentales pour obtenir les intensités correctes.

Deux autres détecteurs étaient introduits après 1955: le compteur proportionnel et le compteur à scintillation composé d'un cristal de NaI couplé à un photomultiplicateur. Dans ces deux compteurs la décharge est limitée en volume, ainsi l'impulsion électronique recueillie est proportionnelle à l'énergie des rayons $\mathrm{X}$ pénétrant dans le compteur. Mais comme l'amplification de l'impulsion est faible il est nécessaire d'amplifier le signal. L'emploi supplémentaire d'un discriminateur d'impulsion permet d'enregistrer uniquement les énergies $\mathrm{X}$ voulues et pas celles correspondant par exemple au bruit de fond. Le temps mort de ces compteurs est très faible, ce qui permet de travailler avec des intensités considérables.

En ce qui concerne les méthodes d'enregistrement, il était possible de balayer un domaine de diffraction angulaire avec le goniomètre, ou d'effectuer des analyses sur un point angulaire fixe afin d'obtenir l'intensité maximale pour une analyse quantitative. On pouvait choisir entre compter pendant un temps fixe en enregistrant le nombre des impulsions, ou choisir un nombre d'impulsions et mesurer le temps nécessaire pour l'atteindre. Pour des raies moins bien définies ou élargies, il était possible de mesurer l'intensité intégrale en effectuant plusieurs balayages du goniomètre sur la région prédéfinie.

Dans le cas d'intensités faibles, il était toujours nécessaire de corriger par le fond spectral. Le temps disponible pour l'analyse totale étant limité, il fallait le déterminer de façon optimale. On peut montrer qu'on arrive à des résultats optimums si $t_{p} / t_{b}=\sqrt{r_{p} / r_{b}}$; où $t_{p}$ et $t_{b}, r_{p}$ et $r_{b}$ sont respectivement, les temps d'enregistrement du pic et du fond spectral, et les intensités 
du pic et du fond spectral. En choisissant les conditions optimales pour la tension, l'anode, les fentes, le détecteur, il faut que $\sqrt{\mathrm{r}_{\mathrm{p}}}-\sqrt{\mathrm{r}_{\mathrm{b}}}$ soit un maximum.

Une autre erreur de mesure est due au fait que seulement un nombre de particules très faible dans l'échantillon peut en réalité contribuer à la diffraction d'une raie. En tournant l'échantillon dans son plan propre, on peut augmenter le nombre de cristallites diffractants. Dans les années suivantes, les erreurs instrumentales ont été fortement réduites, et la statistique des cristallites est aujourd'hui l'erreur la plus importante.

La diffraction des rayons $\mathrm{X}$ a plusieurs applications, la plus importante étant l'identification des phases. Quant à l'analyse quantitative, sans correction de l'absorption des rayons X dans un mélange, on peut seulement doser des modifications de composition chimique. L'exemple classique du mélange d'anatase et de rutile est bien connu. Un autre cas, souvent étudié, est la mesure de l'austénite résiduelle dans les ferrites. Une des premières expériences que nous avions effectuée était l'analyse quantitative de deux modifications de phtanocianide bleu. Il était possible de construire une droite d'étalonnage en mesurant l'intensité intégrale d'une raie.

Dans les premières années il existait très souvent des confusions avec des raies inattendues, par exemple, les raies de diffraction du tungstène (W) provenant de la cathode, ou des raies non tabulées comme Mo K $\beta 5$.

Si on compare le diffractomètre et les chambres photographiques focalisantes, comme celle de Guinier-de Wolf, il faut remarquer que les chambres, enregistrant en -même temps le diagramme, n'avaient pas besoin d'un générateur bien stabilisé, donc meilleur marché, et que la résolution était en général meilleure qu'avec un diffractomètre. D'un autre côté, si on ne mesurait qu'une raie pour une analyse quantitative, les résultats étaient plus précis, parce que la reproductibilité du film photographique est moins bonne.

\section{FLUORESCENCE $X$}

Contrairement à la diffraction, la spectrométrie par fluorescence était une nouvelle technique. Les progrès était dus à l'introduction d'appareils constamment améliorés par les constructeurs et par les développements motivés par des publications et des conférences.

Avantages de cette méthode:

- on peut analyser tous les éléments, sauf les éléments très légers,

- les spectres sont simples, car uniquement les raies résultantes des transitions électroniques internes sont présentes,

- la méthode est non-destructive, on peut répéter une analyse plusieurs fois sur le même échantillon. De cette façon, on peut localiser l'origine des déviations, des erreurs, comme la mise en place de l'échantillon, le positionnement du goniomètre, la stabilité de débit de rayons $\mathrm{X}$.

L'enregistrement des photons caractéristiques est un processus statistique avec une distribution normale. Au début, l'intensité disponible, c'est à dire le nombre de photons pénétrant dans le compteur était assez faible, 1 'erreur statistique était alors l'erreur dominante. Cependant, on pouvait contrôler cette erreur en choisissant le nombre d'impulsions enregistrées.

Les erreurs instrumentales deviennent moins importantes les années suivantes, et ce sont maintenant les erreurs dues à l'échantillonnage qui déterminent la précision d'une analyse. 
Après le travail des précurseurs, Friedrich et Birks, qui ont montré les possibilités de cette méthode, le développement s'effectua suivant deux directions:

- Les sociétés spécialisées en spectroscopie U.V. développaient des analyseurs multicanaux, comme ARL, Hilger,

- celles actives en diffraction ajoutaient un accessoire à leur diffractomètre. Comme la méthode avait besoin d'être connue, l'ajout d'un accessoire était une solution économique pour la tester en pratique.

L'analyse qualitative était effectuée en balayant le domaine angulaire avec le goniomètre et en mesurant successivement les éléments. En modifiant un diffractomètre en spectromètre, on pouvait employer le même générateur, le même goniomètre, la même baie électronique, par contre l'échantillon doit être remplacé par un cristal analyseur et il faut ajouter un tube à rayons $\mathrm{X}$ de spectrométrie et une chambre pour introduire les échantillons.

$\mathrm{Au}$ début, on utilisait un tube de tungstène sous une tension de $60 \mathrm{kV}$, puis des tubes avec d'autres anodes et sous une tension encore plus élevée. Tout d'abord, on supposait que la meilleure excitation consistait à utiliser les rayonnements monochromatiques provenant de l'anode d'énergie très proche de la discontinuité d'absorption des éléments à analyser. Après, il était évident que le spectre primaire blanc était fortement responsable de l'excitation.

Comme le rayonnement émis par des éléments légers est absorbé par l'air, les américains ont placé l'échantillon, le cristal et le compteur Geiger dans une atmosphère d'hélium. En Europe, on a préféré mettre le cristal analyseur et le chemin optique en vide partiel. L'échantillon est irradié par le bas, facilitant la préparation de l'échantillon. Enfin, le faisceau de rayons $\mathrm{X}$ était défini par un système de collimateurs et diffracté par un cristal. Avec cet appareil, il était possible d'analyser les éléments jusqu'au chlore.

Cet appareil assez simple nous a permis de développer quelques principes concernant cette méthode d'analyse par fluorescence $X$. Le compteur GM ne pouvait pas traiter des intensités très importantes, toutefois celles-ci n'étaient pas disponibles en ces années. Il existait seulement quelques cristaux analyseurs, comme le quartz $(2 \mathrm{~d}=0.668 \mathrm{~nm})$ et le $\mathrm{LiF}(2 \mathrm{~d}=$ $0.404 \mathrm{~nm}$ ), tout en se rappelant que plus la valeur de $d$ est petite, meilleur est le pouvoir de résolution pour séparer des raies voisines. Les cristaux et les collimateurs se changeaient manuellement.

Pour une analyse qualitative, on balayait la région angulaire en totalité. Il existait des tables avec les cristaux habituels pour effectuer la conversion des angles mesurés en longueur d'onde. Il était possible de choisir les conditions expérimentales, comme la vitesse du goniomètre et la constante de temps de l'intégrateur pour obtenir un résultat optimum en fonction du temps total disponible pour l'analyse. D'autres tables donnaient les longueurs d'onde caractéristiques pour tous les éléments, et ainsi on pouvait imputer chaque raie à un élément donné.

Pour l'analyse quantitative, on fixait le goniomètre sur la position exacte donnée par les tables, ou à l'aide d'un échantillon étalon. Il était nécessaire de corriger les intensités faibles en tenant compte du fond spectral. On pouvait choisir entre les méthodes à temps fixe ou à nombre de coups fixe. On a rapidement établi que la méthode de temps fixe conduit aux meilleurs résultats et est plus rapide. Des méthodes statistiques nous ont aidé à déterminer les conditions optimales.

La spectrométrie $\mathrm{X}$ était, en ce temps là une méthode relative; le nombre de coups obtenu avec un échantillon inconnu était comparé au nombre mesuré avec un étalon de composition connue. Ces mesures permettaient de construire une courbe d'étalonnage pour chaque élément présent dans le matériau. Quand la méthode fut bien développée, on automatisa l'analyse 
séquentielle et la spectrométrie par fluorescence $X$ devint une méthode de routine, l'analyse qualitative n'était plus nécessaire.

Les instruments s'amélioraient continuellement: des intensités plus élevées étaient obtenues grâce à des cristaux analyseurs plus efficaces, une tension et une puissance plus élevées pour le tube à rayons $\mathrm{X}$, mais il était également indispensable d'augmenter le domaine analytique. L'introduction de deux autres détecteurs offrait cette possibilité: le compteur proportionnel avec une circulation de gaz continu et le compteur à scintillation utilisé pour les éléments moyens et lourds. Les raies caractéristiques des éléments légers sont presque totalement absorbées par l'air. De ce fait, il faut évacuer l'ensemble du chemin optique, y compris le compteur. La fenêtre des compteurs proportionnels scellés est trop épaisse pour laisser traverser les raies des éléments légers. La solution du problème était de développer un détecteur avec une fenêtre très mince mais le gaz pouvant s'échapper, on proposa un écoulement continu du flux gazeux composé d'argon et de méthane. Il restait à régler quelques problèmes techniques comme: la stabilité du flux gazeux, la densité du gaz, sa pureté et l'isolation des connections électriques. La décharge électrique de ces deux compteurs après l'absorption d'un photon est très locale, ce qui conduit à un temps mort très faible permettant de traiter des intensités très élevées. L'impulsion électronique recueillie après l'absorption du photon caractéristique étant d'amplitude très faible, une amplification est nécessaire. Pour les deux compteurs l'impulsion électrique est proportionnelle à l'énergie de photon $\mathrm{X}$ incident et on peut introduire un discriminateur d'impulsions séparant l'énergie caractéristique des signaux gênants comme le bruit de fond ou les raies de deuxième ordre d'éléments plus lourds. Cette opération était effectuée en introduisant une baie électronique auxiliaire entre l'amplificateur et le système de comptage. On peut choisir un niveau inférieur et un niveau supérieur en formant une fenêtre pour ne laisser compter que les photons caractéristiques.

Les longueurs d'onde des éléments légers étant plus longues, une résolution moindre est nécessaire, permettant l'utilisation d'un collimateur avec des espacements larges entre les plaques en plus de celui pour les éléments lourds avec des espacements fins. De même, on utilisa deux cristaux, l'un avec une distance réticulaire faible pour les éléments lourds, l'autre avec une valeur de $\mathrm{d}$ plus élevée pour les éléments légers.

Comme les raies de fluorescence sortent d'une couche très mince de l'échantillon, la surface doit être représentative du volume total. Ainsi pour égaliser les rainures de la surface on fait tourner l'échantillon pendant l'irradiation. Ces premiers "vrais" spectromètres étaient commercialisés après 1958 avec un grand succès. Des milliers de ces systèmes ont été installés dans le monde.

Avec cet instrument, la méthode d'analyse par fluorescence était introduite et devint très populaire, grâce aux travaux effectués par les laboratoires d'application, ainsi qu'aux cours, conférences et démonstrations. Il était en fait nécessaire de vendre la méthode avant de pouvoir vendre le système spécifique. Pour commencer, on avait besoin d'une grande flexibilité pour mener à bien les applications. Ensuite, dès que les problèmes étaient bien définis et réglés, la méthode devint une routine, permettant d'introduire des instruments séquentiels automatiques.

En outre, les appareils multicanaux des années 1965 devenaient plus compacts, avec un nombre de canaux limités, préalignés pour des éléments préchoisis. Chaque canal étant composé d'un collimateur, d'un cristal, d'un détecteur et d'un système de comptage, l'analyse des éléments s'effectue simultanément. Il fallait choisir entre les cristaux plans ou les cristaux courbes, ces derniers donnant une intensité plus grande, en étant plus difficile à aligner. 
L'intensité obtenue, ainsi que la précision augmentaient car chaque partie de l'appareil était améliorée.

D'autres instruments étaient construits dans un but très spécifique comme la mesure d'épaisseur de couches d'étain. Pour déterminer l'épaisseur d'une couche, il est souvent avantageux d'employer une méthode de rapport, par exemple pour déterminer l'épaisseur d'une couche de chrome sur le fer, le rapport des intensités des raies $K \alpha$ sur $K \beta$ est plus sensible que les intensités directes.

Pendant des années nous avons rencontré des cas curieux. Par exemple, la tension du tube à rayons $\mathrm{X}$ est indiquée sur le panneau du générateur. Pour contrôler cette valeur, nous avons enregistré le spectre blanc d'un tube avec une anode de W, diffusé sur un échantillon de matrice légère comme le sucre. Cette méthode peut en outre, être utilisée pour contrôler la pureté du spectre primaire. L'énergie sur le diagramme où l'intensité passe par le zéro doit correspondre à la tension indiquée. Malheureusement ce n'était jamais le cas! Finalement, nous avons réalisé que cette intensité n'était pas due à la diffusion cohérente mais à la diffusion incohérente. Après cette correction, les deux valeurs étaient effectivement en accord. La longueur d'onde mesurée pour un élément peut parfois varier des valeurs théoriques données dans les tables. L'origine de ce phénomène peut être la réfraction ou l'effet chimique. Ainsi, l'étalon utilisé pour aligner le goniomètre sur l'angle correct doit avoir la même composition chimique que l'échantillon. On peut observer cet effet, par exemple avec le $\mathrm{Mg}$, où une déviation angulaire est décelée entre le métal et les oxydes. Par ailleurs, on peut utiliser ces effets pour déterminer la valence des éléments présents dans l'échantillon.

Parfois, on mesurait des raies non indiquées dans les tables, comme le doublet $\mathrm{K} \alpha 3 / \mathrm{K} \alpha 4$, ou des raies modifiées par l'effet Auger.

On peut également obtenir des raies inexplicables à cause des structures cristallographiques des cristaux analyseurs. Pour le topaz, on emploie la raie 333, mais 111 et 222 sont parfois présentes! Le LiF 220 est utilisé pour obtenir une grande résolution, mais dans certains cas on peut observer une raie correspondant aux plans 110, ce qui est interdit par les lois cristallographiques.

Une autre source provoquant une "fausse » raie est la diffraction du spectre blanc par un cristallite assez grand présent dans l'échantillon et qui est par accident dans une position favorable pour être diffracté.

En conclusion, on peut constater qu'après les vingt premières années la fluorescence $\mathrm{X}$ devint une méthode spectrographique bien établie, complémentaire à d'autres méthodes d'analyse chimique. 